\title{
The search for a genetic factor associating with immune restoration disease in HIV patients co-infected with Mycobacterium tuberculosis
}

\author{
Jacquita S. Affandi ${ }^{\mathrm{a}}$, Manoj Kumar ${ }^{\mathrm{b}}$, Upasna Agarwal ${ }^{\mathrm{c}}$, Sarman Singh ${ }^{\mathrm{b}}$ and Patricia Price ${ }^{\mathrm{a}, *}$

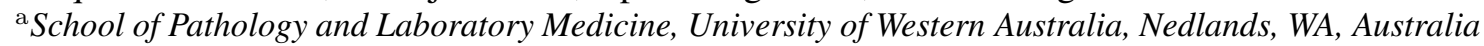 \\ ${ }^{\mathrm{b}}$ Department of Laboratory Medicine, All India Institute of Medical Sciences, New Delhi, India \\ ${ }^{\mathrm{c}}$ Lala Ram Sarup Institute of Tuberculosis and Respiratory Diseases, New Delhi, India
}

\begin{abstract}
.
BACKGROUND: Up to $43 \%$ of HIV-infected patients co-infected with Mycobacterium tuberculosis experience exacerbations of tuberculosis (TB) after commencing antiretroviral therapy (ART). These are termed immune restoration disease (IRD). It is unclear why individual susceptibility varies.

OBJECTIVE: We investigate if single nucleotide polymorphisms (SNP) in genes encoding cytokines, chemokines and their receptors associate with development of an IRD event in patients of two different ethnicities.

METHODS: DNA samples were available from small well-characterised groups of HIV patients treated in Cambodia (TB-IRD, $n=17$; $\mathrm{HIV}^{+} \mathrm{TB}^{+}$controls, $n=55$ ) and India (TB-IRD, $n=19$; $\mathrm{HIV}^{+} \mathrm{TB}^{+}$controls, $n=43$ ). HIV patients with a TB diagnosis but no evidence of IRD were included to control for susceptibility to TB per se. Sixteen SNP implicated in inflammation or mycobacterial disease were genotyped.

RESULTS: Susceptibility to TB-IRD associated with carriage of TNFA-1031*T (rs1799964; $P=0.05$ ) and SLC11A1 D543N*G (rs 17235409; $P=0.04)$ in Cambodian patients and carriage of IL18-607*G (rs 1946518; $P=0.02)$ and VDR FokI $(\mathrm{F} / \mathrm{f}) * \mathrm{~T}(\mathrm{rs} 10735810 ; P=0.05)$ in Indian patients.

CONCLUSIONS: Associations between polymorphisms in immune-related genes and TB-IRD were found, but none were common across two ethnicities.
\end{abstract}

Keywords: TB-IRD susceptibility, immunogenetic factor, single nucleotide polymorphism

\section{Introduction}

Tuberculosis is the most common opportunistic infection associated with HIV and is the leading cause

* Corresponding author: Patricia Price, School of Pathology and Laboratory Medicine, University of Western Australia, Nedlands WA 6009, Australia. Tel.: +61 89224 0378; Fax: +61 89224 0204; E-mail: patricia.price@uwa.edu.au. of morbidity and mortality in people living with HIV/AIDS in resource-limited settings. Combination antiretroviral therapy (ART) has reduced the incidence and severity of Mycobacterium tuberculosis (TB) infections in HIV patients. When they begin ART, a proportion of HIV-TB co-infected patients experience atypical presentations of tuberculosis termed immune restoration disease (TB-IRD). This can occur despite favourable virological responses and increases in $\mathrm{CD}^{+}{ }^{+}$T-cell counts on ART $[8,10]$. TB-IRD is com- 
mon in developing countries where TB is endemic such as Cambodia and India [2,4]. We have sought common disease-associated polymorphisms by investigating genetic associations in patients of different ethnicities from the developing world. Samples were available from HIV patients from Cambodia and India who presented with TB-IRD, as well as HIV-positive TBpositive control patients.

Single nucleotide polymorphisms (SNP) affecting cytokines, chemokines and their receptors may influence development of an IRD event via effects on HIV disease, on TB itself or on the response to ART [10]. We evaluated single nucleotide polymorphisms (SNP) in inflammatory and other immune-related genes selected based on a literature search for laboratory evidence of functional consequences for an allele and associations with autoimmune disorders, infectious diseases, respiratory and mycobacterial diseases [1]. Here we present the associations of immune-related genotypes on the risk of developing IRD in TB patients. A significant association places this SNP or one in linkage disequilibrium in the path to disease. If the association is similar in two ethnicities, one can argue that the SNP is biologically important and where differences are observed between ethnicities, one can argue that the effect is random or that the haplotypic associations around the SNP vary with ethnicity.

\section{Methods and materials}

DNA samples were available from two prospective cohort studies of HIV patients beginning ART with known TB status. Patients were monitored for 612 months to establish the occurrence of IRD using guidelines by the treating clinicians as proposed by the International Network for the Study of HIV-associated IRIS [8]. Both studies were approved by Human Research Ethics Committees of the relevant institutions and all participants provided written informed consent.

1. $\mathrm{HIV}^{+} \mathrm{TB}^{+}$patients were recruited from the National Centre for HIV/AIDS, Dermatology, and Sexually Transmitted Disease, Social Health Clinic, in Phnom Penh, Cambodia [4]. These included 10 patients with paradoxical TB-IRD (ie: whilst on TB treatment) and 7 with ARTassociated TB-IRD (ie: a new diagnosis of TB on ART) [total TB-IRD patients $(n=17)$ and $\mathrm{HIV}^{+}$ $\mathrm{TB}^{+}$controls $\left.(n=55)\right]$.
2. TB-IRD patients ( $n=19$; all paradoxical TBIRD) and $\mathrm{HIV}^{+} \mathrm{TB}^{+}$controls $(n=43)$ were recruited at the Lala Ram Sarup (LRS) TB and Respiratory Diseases (Delhi, India) after initiating TB therapy $[2,13]$.

DNA samples were extracted from PBMC or saliva using a QIAamp DNA Blood Mini Kit (QIAGEN, Valencia, CA, USA) and stored at $-80^{\circ} \mathrm{C}$. Sixteen SNPs were genotyped using TaqMan FAM or VIClabelled probes and Universal PCR Master Mix (Applied Biosystems, Foster City, CA, USA). BAT1 intron 10 (rs9281523) was genotyped using a custom TaqMan assay [17]. DNA samples were diluted to $10 \mathrm{ng} / \mu \mathrm{L}$ and amplified on an ABI Prism 7900 HT (Applied Biosystems) or Roche LC480 (Roche Applied Science, Indianapolis, IL, USA) in 384-well microtiter plates. Thermocycling involved one step of $10 \mathrm{~min}$ at $95^{\circ} \mathrm{C}$, followed by 40 cycles of denaturation at $92^{\circ} \mathrm{C}$ for $15 \mathrm{~s}$ with annealing and extension at $60^{\circ} \mathrm{C}$ for $1 \mathrm{~min}$. Fluorescence was read on an ABI 7900 Sequence Detector (Applied Biosystems) or Roche LC480 (Roche Applied Science) using proprietary allelic discrimination software. Repeats and non-template controls were included in each run. The median (range) genotyping success rate was $99 \%$ (98-100\%) for each SNP in both cohorts. Alleles are recorded as "gene-nucleotide position*allele 1/allele 2", with allele 1 being the most frequent allele in Caucasians (previously defined as wildtype). In accordance with our earlier publications [15, 16], TNF polymorphisms are described by their "traditional" names, TNF-308 and TNF-1031. We note that they lie 307 and 1030 bases (resp.) from the transcription start site [14].

Allele and genotype frequencies in each ethnic group conformed with Hardy-Weinberg equilibrium (HWE) when assessed using GENEPOP v3.3 [11]. Fisher's exact test was used to identify factors associated with risk of developing TB-IRD. Results are presented as Odds ratios (OR), their $95 \%$ confidence intervals (95\% CI) and $P$ values, using the homozygous wild-type allele as the reference group. $P \leqslant 0.05$ was accepted as indicating a significant difference. Analyses were performed using GraphPad (Prism version 5.02 for Windows, GraphPad Software, San Diego, CA, USA). Logistic regression analyses utilised Stata (StataCorp. 2009, Stata Statistical Software: Release 11. College Station, Texas, USA).

\section{Results}

A logistic regression that included all SNP, both cohorts and CD4T-cell counts (square root transformed 
Table 1

TB-IRD associate with alleles of TNFA-1031 and SLC11A1 D543N in Cambodian patients and VDR FokI (F/f) and IL18-607 in Indian patients

\begin{tabular}{|c|c|c|c|c|c|c|c|c|c|c|c|c|}
\hline & \multirow[b]{2}{*}{ WT/var } & \multirow[b]{2}{*}{ SNP rs no. } & \multicolumn{5}{|c|}{ Cambodia } & \multicolumn{5}{|c|}{ India } \\
\hline & & & $\begin{array}{c}\mathrm{HIV}^{+} \mathrm{TB}^{+} \\
(n=55)\end{array}$ & $\begin{array}{l}\text { TB-IRD } \\
(n=17)\end{array}$ & $95 \% \mathrm{CI}$ & OR & $P$ & $\begin{array}{c}\mathrm{HIV}^{+} \mathrm{TB}^{+} \\
(n=43)\end{array}$ & $\begin{array}{l}\text { TB-IRD } \\
(n=19)\end{array}$ & $95 \% \mathrm{CI}$ & OR & $P$ \\
\hline \multicolumn{3}{|l|}{ Male/Female } & $38 / 17$ & $11 / 6$ & & & & $33 / 10$ & $16 / 3$ & & & \\
\hline \multicolumn{3}{|l|}{ Age (years) ${ }^{\mathrm{a}}$} & $33(25-63)$ & $36(26-45)$ & & & & $35(18-65)$ & $36(18-51)$ & & & \\
\hline \multicolumn{3}{|c|}{ Baseline CD4 ${ }^{+} \mathrm{T}$ cells $/ \mu \mathrm{L}^{\mathrm{a}}$} & $62(2-239)$ & $45(1-227)$ & & & & $100(2-198)$ & $78(14-189)$ & & & \\
\hline \multirow{2}{*}{\multicolumn{3}{|c|}{ Time between ART and IRD (days) ${ }^{\mathrm{a}}$}} & $N / A$ & $10(7-89)^{\mathrm{e}}$ & & & & $N / A$ & $17(5-109)^{\mathrm{e}}$ & & & \\
\hline & & & & $10(1-28)^{\mathrm{t}}$ & & & & & & & & \\
\hline TNFA-308 & G/A & 1800629 & $22^{\circ}$ & $25^{\circ}$ & $0.3-4.3$ & 1.2 & $1^{\mathrm{a}}$ & $12^{\mathrm{c}}$ & $16^{c}$ & $0.3-6.7$ & 1.4 & $0.69^{\mathrm{a}}$ \\
\hline TNFA-1031 & $\mathrm{C} / \mathrm{T}$ & 1799964 & 47 & 77 & $1.0-12$ & 3.6 & 0.05 & 63 & 47 & $0.2-1.6$ & 0.53 & 0.28 \\
\hline BAT1 (intron 10) & Ins $\mathrm{C}$ & 9281523 & 22 & 31 & $0.5-5.5$ & 1.6 & 0.51 & 7 & 10 & $0.2-10$ & 1.5 & 0.64 \\
\hline IL1A + 4845 & C/A & 17561 & 9 & 33 & $0.9-25$ & 4.8 & 0.08 & 60 & 63 & $0.4-3.6$ & 1.2 & 1 \\
\hline IL2-330 & $\mathrm{T} / \mathrm{G}$ & 2069762 & 62 & 37 & $0.1-1.2$ & 0.37 & 0.09 & 72 & 79 & $0.4-5.3$ & 1.5 & 0.76 \\
\hline IL4-589 & $\mathrm{C} / \mathrm{T}$ & 2243250 & 50 & 59 & $0.5-4.3$ & 1.4 & 0.58 & 30 & 26 & $0.3-2.8$ & 0.82 & 1 \\
\hline IL10-1082 & $\mathrm{A} / \mathrm{G}$ & 1800896 & 13 & 12 & $0.2-4.7$ & 0.88 & 1 & 44 & 42 & $0.3-2.7$ & 0.92 & 1 \\
\hline IL12B 3'UTR & $\mathrm{A} / \mathrm{C}$ & 3212227 & 75 & 65 & $0.2-2$ & 0.63 & 0.54 & 67 & 61 & $0.3-2.5$ & 0.79 & 0.77 \\
\hline IL18-137 & $\mathrm{G} / \mathrm{C}$ & 187238 & 30 & 35 & $0.4-4$ & 1.3 & 0.77 & 31 & 42 & $0.5-5$ & 1.6 & 0.4 \\
\hline IL18-607 & $\mathrm{T} / \mathrm{G}$ & 1946518 & 59 & 76 & $0.6-7.8$ & 2.2 & 0.26 & 31 & 63 & $1.2-12$ & 3.8 & 0.02 \\
\hline CCL2-2518 & $\mathrm{A} / \mathrm{G}$ & 1024611 & 73 & 71 & $0.2-3.3$ & 0.9 & 1 & 49 & 58 & $0.5-4.3$ & 1.4 & 0.59 \\
\hline VDR FokI (F/f) & $\mathrm{C} / \mathrm{T}$ & 10735810 & 67 & 76 & $0.5-5.7$ & 1.6 & 0.55 & 40 & 68 & $1.1-10$ & 3.3 & 0.05 \\
\hline SLC11A1 D543N & G/A & 17235409 & 39 & 12 & $0.04-1.0$ & 0.21 & 0.04 & 9 & 5 & $0.06-5.2$ & 0.54 & 1 \\
\hline CD14 C(-159)T & $\mathrm{C} / \mathrm{T}$ & 2569190 & 66 & 71 & $0.4-4.7$ & 1.3 & 1 & 72 & 63 & $0.2-2.1$ & 0.65 & 0.55 \\
\hline IL28B - SNP1 & $\mathrm{T} / \mathrm{G}$ & 8099917 & 24 & 12 & $0.08-2.1$ & 0.42 & 0.5 & 30 & 16 & $0.1-1.7$ & 0.43 & 0.35 \\
\hline IL28B - SNP2 & $\mathrm{C} / \mathrm{T}$ & 12979860 & 26 & 6 & $0.02-1.5$ & 0.18 & 0.09 & 37 & 47 & $0.5-4.4$ & 1.5 & 0.58 \\
\hline
\end{tabular}

$95 \%$ CI $=95 \%$ Confidence interval; OR = odds ratio; SNP $=$ single nucleotide polymorphism. Significant $P$ values $<0.05$ indicated in bold.

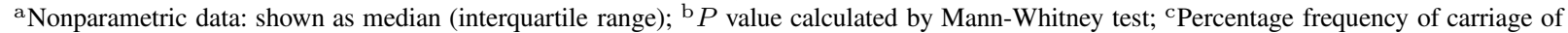

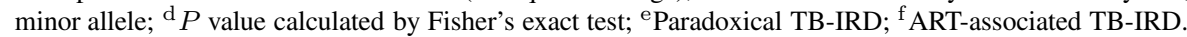

to approximate a normal distribution) generated significant associations with heterozygosity at IL1A +4845 $(p=0.05)$ and IL18-607 ( $p=0.014)$. Sequential removal of SNP that were not associated with TB-IRD yielded a significant fit $(p=0.0175)$ with a model including heterozygosity at SLC11 A1 D543 N ( $p=$ $0.036)$ and IL18-607 $(p=0.026)$. However these analyses do not reveal ethnic differences, so we present simple bivariate associations (Table 1).

In Cambodian patients, carriage of allele $\mathrm{T}$ in TNFA-1031 (rs1799964) and allele G in SLC11A1 D543 N (rs17235409) $[P=0.05, \mathrm{OR}=3.6 ; P=0.04$, $\mathrm{OR}=0.21$; resp] was associated with susceptibility to TB-IRD. Trends were observed with carriage of allele A in IL1A +4845 and allele T in IL2-330 $(P=0.08$ and $P=0.09$ resp). In Indian TB patients, carriage of allele G in IL18-607 and allele T in VDR FokI (F/f) was associated with increased incidence of susceptibility to TB-IRD $(P=0.02, \mathrm{OR}=3.8 ; P=0.05, \mathrm{OR}=$ 3.3 , resp).

\section{Discussion}

The results show distinct patterns of association in the two populations. For example; carriage of the vari- ant allele in SLC11A1 D543N conferred protection from an IRD event in Cambodians but there was no effect in Indian patients. This SNP has been associated with susceptibility to inflammatory diseases such as Crohn's disease in Greek patients [5] and Buruli ulcers in Ghanaian patients [12]. Carriage of the variant allele in IL18-607 conferred an almost 4-fold increased risk of developing an IRD event in Indian patients, but had no effect in Cambodians. No such association was observed in a cohort of HIV-negative Indian pulmonary TB patients [7], but in a Han Chinese pulmonary TB cohort without HIV, carriage of the variant allele in IL18-607 was associated with a decreased risk of developing TB [6].

Ethnic differences may arise when the SNP assayed is not directly responsible for the effect, but lies in a haplotype that is differentially carried in donors of each ethnicity. This cannot be determined for ILI8 and SLC11A1 at this time, but there is more information about TNF. Carriage of the variant allele in TNFA-1031 (rs1799964) in TB-IRD patients associated with a 4-fold greater risk in Cambodians, but this was not evident in the Indian cohort. TNFA-1031*2 affects neuropathy in Malay and Chinese HIV-infected patients [3] and haplotype blocks carrying TNF-1031 differ in frequency between Indian, Chinese and Cau- 
casian donors [16]. TNFA-308*2 and TNFA-1031*2 lie in distinct "TNF block haplotypes". A common disease associated haplotype incorporating TNFA-308 (rs1800629) is marked by a SNP in the BATl gene and is associated with venous leg ulcers and sepsis in Caucasians [17,18]. TNF-308*2 was not found in a small cohort of Australian HIV patients with IRD associated with non-tuberculous mycobacteria, but was present in 24-34\% of controls ( $p=0.015-0.035$ ) [9]. The haplotype incorporating allele 2 at TNFA-308 and BAT1(int10) is present in Asians [16], so there is a clear dissociation between the role of TNF haplotypes in IRD associated with TB in the developing world (Table 1) and non-tuberculous mycobacteria in Australia [9].

Overall there were associations between polymorphisms in immune-related genes and TB-IRD, but none held in both ethnicities. Although the cohorts were small, it can be argued that effects strong enough to have a use in clinical practise should have emerged. Subsequent studies should investigate patterns of linkage disequilibrium to determine whether the associations observed here reflect ethnic differences in the haplotypic structures across critical genes.

\section{Acknowledgements}

The authors thank Julian Elliott from The Burnet Institute, Melbourne, Victoria and Vonthanak Saphonn and Mean Chhi Vun from National Centre for HIV/ AIDS, Dermatology and Sexually Transmitted Diseases, Ministry of Health, and National Institute of Public Health, Phnom Penh, Cambodia. The authors thank Sally Burrows from the School of Medicine and Pharmacology, University of Western Australia, Perth, Australia, for the multivariable statistical analyses. The authors also thank all patients and controls who donated samples for this study.

\section{Conflict of interest}

The authors have no conflicts of interest to declare.

\section{References}

[1] J.S. Affandi, P. Price and G. Waterer, Can immunogenetics illuminate the diverse manifestations of respiratory infections?, Ther Adv Respir Dis 4 (2010), 161-176.
[2] U. Agarwal, A. Kumar, D. Behera, M.A. French and P. Price, Tuberculosis associated immune reconstitution inflammatory syndrome in patients infected with HIV: meningitis a potentially life threatening manifestation, AIDS Res Ther 9 (2012), 17.

[3] C.S. Chew, C.L. Cherry, D. Imran, E. Yunihastuti, A. Kamarulzaman, S. Varna, R. Ismail, M. Phipps, Z. Aghafar, I. Gut and P. Price, Tumour necrosis factor haplotypes associated with sensory neuropathy in Asian and Caucasian human immunodeficiency virus patients, Tissue Antigens 77 (2010), 126-130.

[4] J.H. Elliott, K. Vohith, S. Saramony, C. Savuth, C. Dara, C. Sarim, S. Huffam, R. Oelrichs, P. Sophea, V. Saphonn, J. Kaldor, D.A. Cooper, M. Chhi Vun and M.A. French, Immunopathogenesis and diagnosis of tuberculosis and tuberculosis-associated immune reconstitution inflammatory syndrome during early antiretroviral therapy, Journal of Infectious Diseases 200 (2009), 1736-1745.

[5] M. Gazouli, V. Atsaves, G. Mantzaris, M. Economou, G. Nasioulas, K. Evangelou, A.J. Archimandritis and N.P. Anagnou, Role of functional polymorphisms of NRAMP1 gene for the development of Crohn's disease, Inflamm Bowel Dis 14 (2008), 1323-1330.

[6] M. Han, J. Yue, Y.Y. Lian, Y.L. Zhao, H.X. Wang and L.R. Liu, Relationship between single nucleotide polymorphism of interleukin-18 and susceptibility to pulmonary tuberculosis in the Chinese Han population, Microbiol Immunol 55 (2011), 388-393.

[7] M. Harishankar, P. Selvaraj, D.N. Rajeswari, S.P. Anand and P.R. Narayanan, Promoter polymorphism of IL-18 gene in pulmonary tuberculosis in South Indian population, Int J Immunogenet 34 (2007), 317-320.

[8] G. Meintjes, S.D. Lawn, F. Scano, G. Maartens, M.A. French, W. Worodria, J.H. Elliott, D. Murdoch, R.J. Wilkinson, C. Seyler, L. John, M.S. van der Loeff, P. Reiss, L. Lynen, E.N. Janoff, C. Gilks and R. Colebunders, Tuberculosis-associated immune reconstitution inflammatory syndrome: case definitions for use in resource-limited settings, Lancet Infect Dis $\mathbf{8}$ (2008), 516-523.

[9] P. Price, G. Morahan, D. Huang, E. Stone, K.Y. Cheong, A. Castley, M. Rodgers, M.Q. McIntyre, L.J. Abraham and M.A. French, Polymorphisms in cytokine genes define subpopulations of HIV-1 patients who experienced immune restoration diseases, AIDS 16 (2002), 2043-2047.

[10] P. Price, D.M. Murdoch, U. Agarwal, S.R. Lewin, J.H. Elliott and M.A. French, Immune restoration diseases reflect diverse immunopathological mechanisms, Clin Microbiol Rev 22 (2009), 651-663.

[11] F. Rousset and M. Raymond, Testing heterozygote excess and deficiency, Genetics 140 (1995), 1413-1419.

[12] Y. Stienstra, T.S. van der Werf, E. Oosterom, I.M. Nolte, W.T. van der Graaf, S. Etuaful, P.L. Raghunathan, E.A. Whitney, E.O. Ampadu, K. Asamoa, E.Y. Klutse, G.J. te Meerman, J.W. Tappero, D.A. Ashford and G. van der Steege, Susceptibility to Buruli ulcer is associated with the SLC11A1 (NRAMP1) D543N polymorphism, Genes Immun 7 (2006), 185-189.

[13] H.R. Sumatoh, B.G. Oliver, M. Kumar, J.H. Elliott, S. Vonthanak, M.C. Vun, S. Singh, U. Agarwal, A. Kumar, H.Y. Tan, A. Kamarulzaman, E. Yunihastuti, H. Saraswati and P. Price, Mycobacterial antibody levels and immune restoration disease in HIV patients treated in South East Asia, Biomark Med 5 (2011), 847-853.

[14] A.M. Uglialoro, D. Turbay, P.A. Pesavento, J.C. Delgado, F.E. McKenzie, J.G. Gribben, D. Hartl, E.J. Yunis and A.E. Gold- 
feld, Identification of three new single nucleotide polymorphisms in the human tumor necrosis factor-alpha gene promoter, Tissue Antigens 52 (1998), 359-367.

[15] F.P. Valente, C. Tan, M. Phipps, C.S. Witt, G. Kaur, I. Gut, R. Allcock and P. Price, TNF block haplotypes associated with conserved MHC haplotypes in European, Asian and Australian Aboriginal donors, Tissue Antigens 74 (2009),

[16] F.P. Valente, C.R.T. Tan, S.E. Temple, M. Phipps, C.S. Witt, G. Kaur, I. Gut, S. McGinn, R.J.N. Allcock, C.S.N. Chew and P. Price, The evolution and diversity of TNF block haplotypes in European, Asian and Australian Aboriginal populations,
Genes \& Immunity 10 (2009), 607-615.

[17] H.J. Wallace, Y.K. Vandongen and M.C. Stacey, Tumor necrosis factor-alpha gene polymorphism associated with increased susceptibility to venous leg ulceration, Journal of Investigative Dermatology 126 (2006), 921-925.

[18] G.W. Waterer, M.W. Quasney, R.M. Cantor and R.G. Wunderink, Septic Shock and Respiratory Failure in Communityacquired Pneumonia Have Different TNF Polymorphism Associations, Am J Respir Crit Care Med 163 (2001), 15991604. 


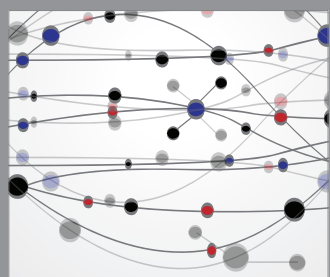

The Scientific World Journal
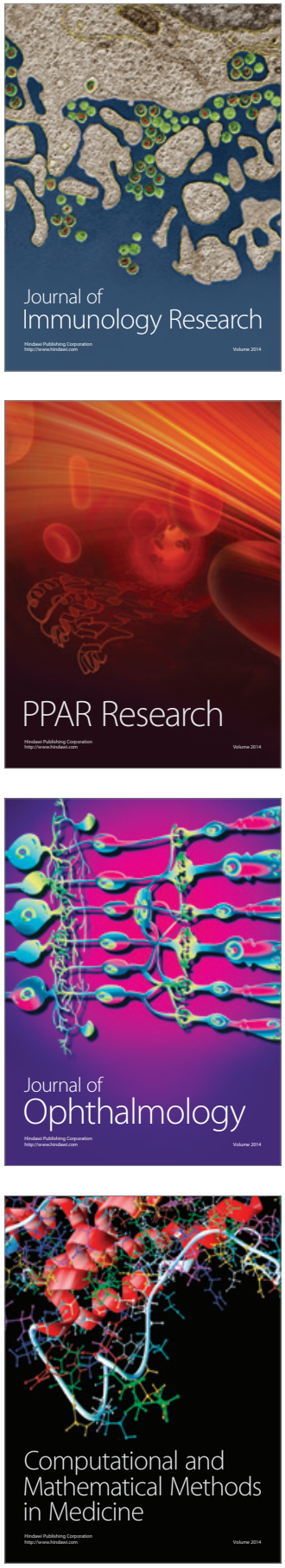

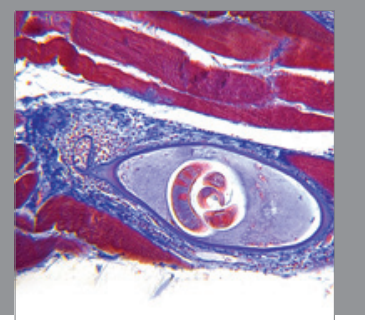

Gastroenterology

Research and Practice
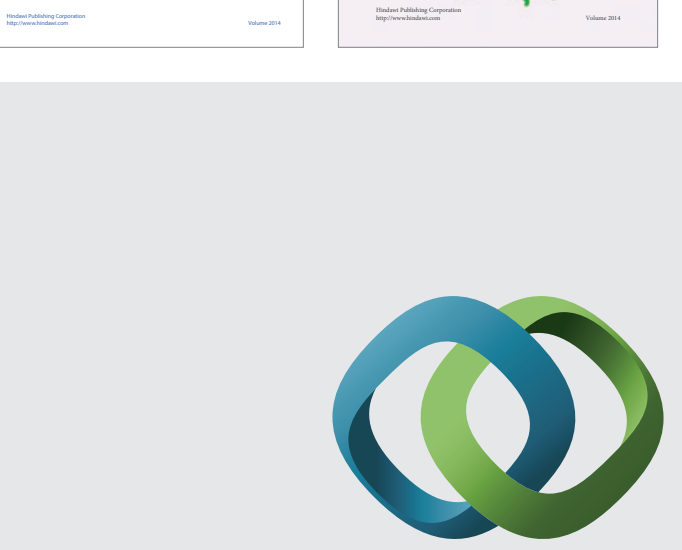

\section{Hindawi}

Submit your manuscripts at

http://www.hindawi.com
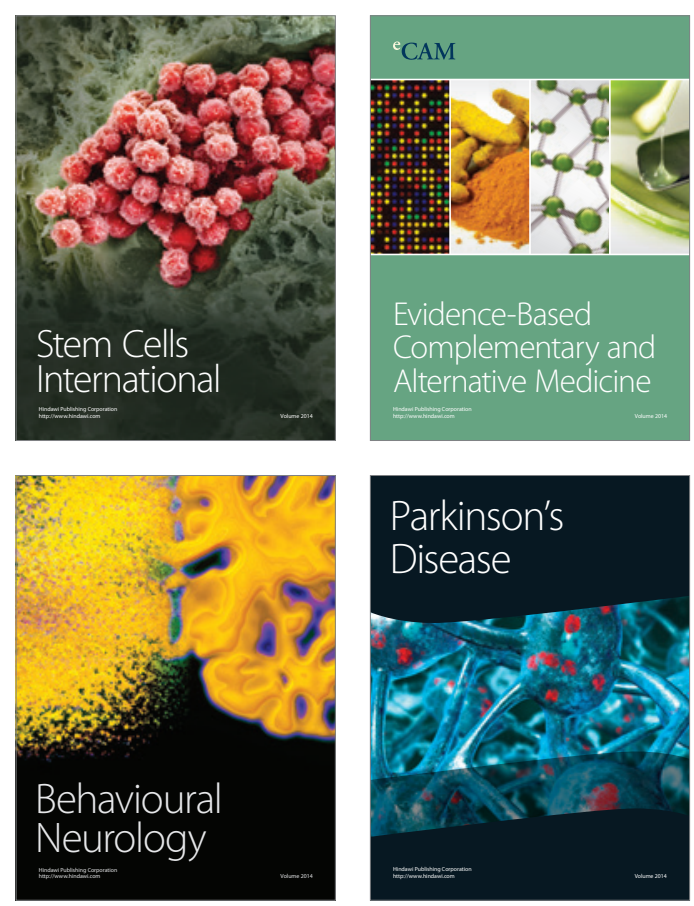

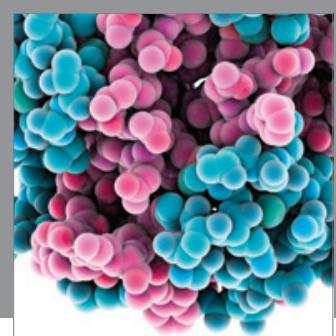

Journal of
Diabetes Research

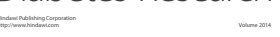

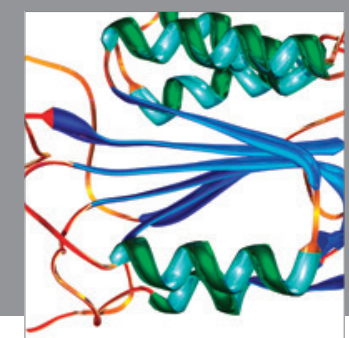

Disease Markers
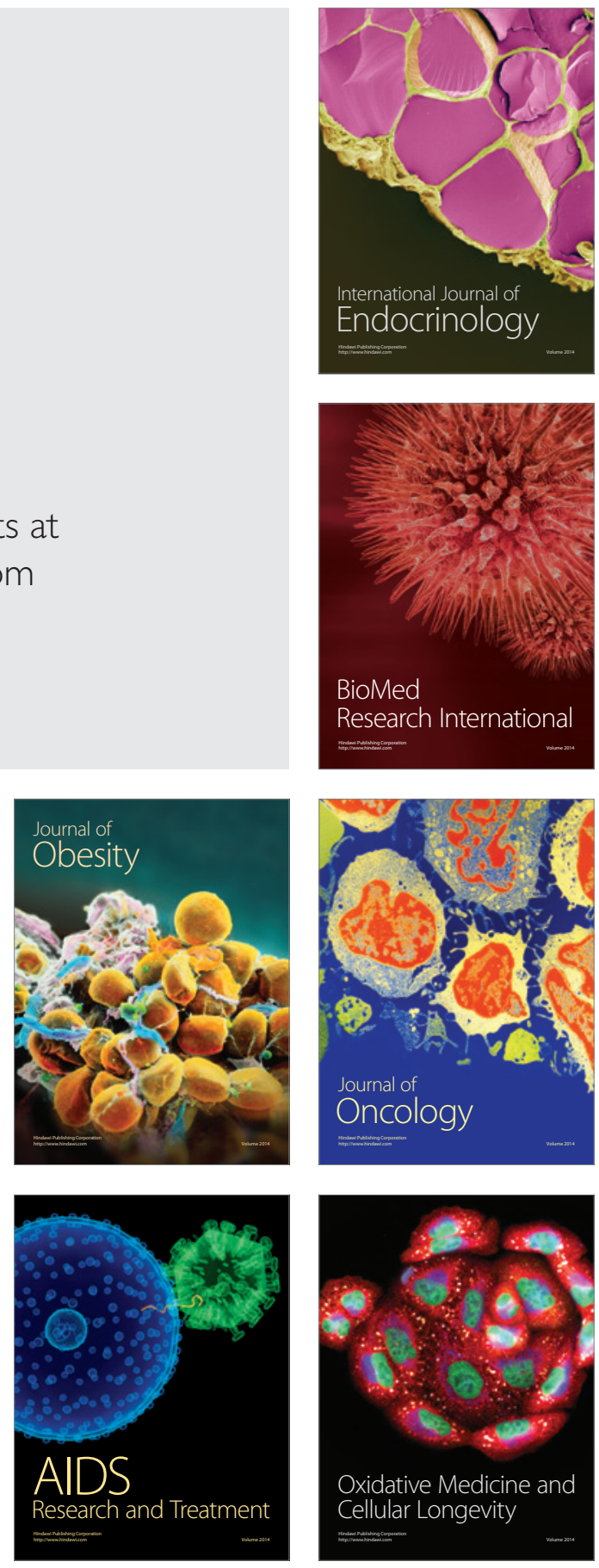